\title{
The potential application of stem cell in dentistry
}

\author{
Ketut Suardita \\ Department of Conservative Dentistry \\ Faculty of Dentistry Airlangga University \\ Surabaya - Indonesia
}

\begin{abstract}
Stem cells are generally defined as cells that have the capacity to self-renewal and differentiate to specialize cell. There are two kinds of stem cell, embryonic stem cell and adult stem cells. Stem cell therapy has been used to treat diseases including Parkinson's and Alzheimer's diseases, spinal cord injury, stroke, burns, heart diseases, diabetes, osteoarthritis, and rheumatoid arthritis. Stem cells were found in dental pulp, periodontal ligament, and alveolar bone marrow. Because of their potential in medical therapy, stem cells were used to regenerate lost or damage teeth and periodontal structures. This article discusses the potential application of stem cells for dental field.
\end{abstract}

Key words: embryonic stem cell, adult stem cells, dentistry

Correspondence: Ketut Suardita, c/o: Bagian Konservasi Gigi, Fakultas Kedokteran Gigi Universitas Airlangga. Jln. Mayjend. Prof. Dr. Moestopo 47 Surabaya 60132, Indonesia.

\section{INTRODUCTION}

The inability of most tissues and organs to repair and regenerate after damage is a problem in medical and dentistry that should be solve. To repair or regenerate damage tissues and organs, many materials and devices were used, but the results are not good. Many complications including infection, inflammation, impaired function, and loosening were happen. These conditions make opportunities to scientist improved therapies.

In medical therapy, stem cells have been used for engineering many tissues and organs. Stem cell researches are interesting knowledge about how to regenerate healthy cells, tissues, and organs from a single cell. Stem cell is a multipotent cell, which can proliferate and differentiate to specific cell. These cells have the capacity to form many different tissue types. Stem cell therapy has been used to treat diseases including Parkinson's and Alzheimer's diseases, spinal cord injury, stroke, burns, heart diseases, diabetes, osteoarthritis, and rheumatoid arthritis. ${ }^{1}$

Regeneration of damage periodontal tissue, bone, pulp, and dentin are problems that the dentist should solve. At present, some traditional approaches are used to repair damage dental tissues. Direct pulp capping using calcium hydroxide is a conventional technique to repair the damage of tooth pulp. Demineralized bone graft was used in order to repair fractured bone. Furthermore, guided tissue regeneration (GTR) and growth factor, for example bone morphogenic proteins (BMPs) were used to regenerate new periodontal tissues. In fact, it is difficult to predict the result of treatments stated above. ${ }^{2}$ Since, stem cells were used to regenerate damage tissue in medical therapy successfully; it is possible that the dentist use stem cell to regenerate lost or damage dental and periodontal structures.
The purpose of this article is to describe and discuss stem cell potential application in dentistry. In future, stem cell therapy will enable new dental treatments for caries, endodontic, periodontal and oral-maxillo facial surgery, alveolar ridge augmentation, and cartilage in the temporomandibular joint.

\section{What is stem cell?}

Stem cell can be described as an immature or undifferentiated cell that is capable of producing an identical daughter cell. Stem cell has two important characteristics that distinguish them from other types of cells. First, they are unspecialized cells that renew themselves for long periods through cell division. The second is that under certain physiologic or experimental conditions, they can be induced to become cells with special functions. Stem cell self-renewal may be perpetuated over many generations, resulting in considerable amplification of stem cell numbers. A stem cell is able to produce at least one type of highly differentiated cell. In traditional thinking, stem cells have been generally recognized as undifferentiated cells with varying degrees of potency. There are three basic measures of stem cell potency i.e. totipotent, pluripotent, and multipotent. ${ }^{3}$

Stem cell has been identified in two kinds of tissues that are in adult tissues, so-called adult stem cell and in embryo, called embryonic stem cell. In a blastocyst of a developing embryo, stem cells differentiate into all of the specialized embryonic tissues. In adult organisms, stem cells act as a repair system for the body; replenishing specialized cells. ${ }^{4}$ Generally adult stem cells present a more limited range of differentiated lineages. Compared to embryonic stem cells, adult stem cell are preferable for therapeutic purposes since they are considered safer for implantation, with lesser 
proliferation capacity and tumorogenecity. Adult stem cells are also easier to differentiate to specific lineages..$^{5}$

\section{Source of stem cell}

Embryonic stem cells are isolated from the inner cell mass of the preimplantation blastocyst and have been derived from mice, non-human primates, and human. They are pluripotent cells, retaining the capacity to generate any and all fetal and adult cell types in vivo and in vitro. By manipulating the culture conditions under which embryonic stem cell differentiate, it has been possible to control and restrict the differentiation pathways. Embryonic stem cell, especially mouse embryonic stem cell have been used to generate a range of distinct phenotypes including haematopoietic precursors, neural cells, adipocytes, muscle cells, myocytes, chondrocytes, pancreatic islet, and osteoblasts in vitro. Because of their capability to differentiate into many different cell types, embryonic stem cell have been recognized as a valuable model system for studying the mechanisms underlying lineage specification during the early stages of mammalian development. 6,7

Stem cell can be identified in many adult mammalian tissues. In some tissues, such as epithelia, blood, and germ line, stem cells contribute to replenishment of cells lost through normal cellular senescence or injury. Stem cells may also be present in other adult organs, such as the brain and pancreas, which normally undergo very limited cellular regeneration or turnover. Adult stem cells are found in specific niches or tissue compartment including skin, liver, intestine, brain, skeletal muscle, myocardium, fatty tissue, and bone marrow. ${ }^{8}$ Bone marrow contains hematopoetic stem cells, which differentiate into every type of mature blood cell; endothelial cell progenitors; and marrow stromal cells, also called mesenchymal stem cells (MSC). MSC can fabricate a spectrum of specializes mesenchymal tissues including bone, cartilage, muscle, marrow stroma, tendon, ligament, fat, and variety other connective tissues.9,10,11

In oral environment, stem cells were isolated from adult dental pulp tissues, periodontal ligament and alveolar bone marrow. ${ }^{12,13,14,15}$ There was an evidence that remnant dental pulp derived from exfoliated deciduous teeth contains a multipotent stem-cell population. ${ }^{16}$

\section{Application of stem cell for medical and dental therapies}

Because of their abilities of unlimited expansion and pluripotency, embryonic stem cells are a potential source for regenerative medicine and tissue replacement after injury or disease. To date, no approved medical treatments have been derived from embryonic stem cell research. Controversies surrounding the legal and moral status of human embryos and the use of embryonic stem cells encompass fundamental issues such as contraception, abortion, the definition of human life, and the rights and legal status of an embryo.

The use of adult stem cells in research and therapy is not as controversial as embryonic stem cells, because the production of adult stem cells does require the destruction of an embryo. Adult stem cells have been proven clinically useful because they can be isolated, transplanted, and effectively reconstitute the damage tissues. Using autologous MSCs dispersed in a collagen-type I gel, Wakitani et al. ${ }^{17}$ succeeded in repairing full-thickness defects on the weight-bearing surface of medial femoral condyles. Furthermore, treatment of MSC with synthetic glucocorticoid dexamethasone stimulates MSC proliferation and support osteogenic lineage differentiated. ${ }^{18}$

Adult stem cells have been used to repairing or regenerating tissues because the limitation of adult cell for tissue regeneration. Grande et al. ${ }^{19}$ reported that autologous chondrocyte cultures could be utilized to repair articular cartilage defects in the rabbit knee. Subsequently, this technique has been applied to the clinical treatment of articular cartilage defects. Although repairing the defect with chondrocytes is attractive, there are limitations related to the harvesting of chondrocytes and expanded these cells. We need large amount of biopsy to get enough cells for transplantations.

In addition to the well-established bone and cartilage lineages, the induction of MSC differentiation into other connective tissues, such as muscle, tendons, and ligaments is also being investigated. For a tissue-engineering approach, marrow-derived MSCs have been used for Achilles tendon repair. MSCs seeded onto a collagen-type I construct incorporated into healing tendons. These MSCs-loaded scaffolds had better alignment of cells and collagen fibers and were more similar to the native tendon than unloaded controls. ${ }^{20}$ Recently, some groups have been examined the treatment of myocardial infarction by application of autologous MSCs in the pig model, and these studies show engraftment, differentiation, and improved function in animals treated with autologous marrow MSCs. ${ }^{21}$

In periodontal treatment, bone marrow-derived mesenchymal stem cells were transplanted to experimental Class III periodontal defects. The aim of this research was to elucidate the behavior of transplanted MSCs in periodontal defects. Four weeks after transplantation, the periodontal defects were almost regenerated with periodontal tissue. ${ }^{22}$

\section{DISCUSSION}

Caries, pulpitis, apical periodontitis and another craniofacial diseases increase health costs and attendant loss of economic productivity. They ultimately result in premature tooth loss and therefore diminishing the quality of life. Within the next few decades, changes in the methods and materials used to treat dental disease will take place. Tissue engineering is a new concept that might be solves the problem in craniofacial regeneration. Tissue engineering is the science of design and manufacture of new tissues to replace damage tissues because of diseases and trauma. The three key elements of tissue engineering are signal for morphogenesis, stem cells for responding to morphogens, and the scaffold of extra cellular matrix.

Stem cells are generally defined as cells that have the 
capacity to self-renewal and differentiate to specialize cell. Stem cells are present in small numbers in many vertebrate adult and fetal tissues. They are responsible for tissue renewal and for regeneration of damaged tissues. During wound healing, dental pulp stem cells have the potential to proliferate and to differentiate into odontoblasts to form dentin. ${ }^{23}$ In the other hand, stem cell derived from periodontal ligament may migrate into periodontal defect, proliferate, and differentiate. ${ }^{24}$

The ability of high expansion and multipotent of differentiation make stem cells are the cell sources for potential therapeutic use and tissue engineering in dentistry. Several studies indicated that stem cells are present in dental pulp, alveolar bone and in the periodontal ligament. ${ }^{12,13,14}$

In endodontic and conservative dentistry, to restore and regenerate the dentin-pulp complex is a problem that very difficult to solve. Direct pulp capping using calcium hydroxide cannot induce new dentin regeneration when there are no odontoblasts remain in dental pulp. Stemcell based tissue engineering may be a new technique to regenerate dentin-pulp complex. Using methodology developed to isolate and characterize mesenchymal stem cells, clonogenic and highly proliferative dental pulp stem cells (DPSCs) have been isolated from adult human teeth. These stem cells maintained their high rate of proliferation even after extensive sub culturing and generated s dentin/ pulp-like complex. Furthermore, it is noteworthy that the amount of dentin and pulp-like tissue formed in transplant far exceeds the amount that would be generated in situ during the lifetime of an organism. Consequently, there is a great potential for the isolation of a large number of DPSCs from a single tooth that could be used for dentinal repair of a number of teeth. ${ }^{12}$ In addition, Iohara et al..$^{25}$ in their research stated that the autogenous transplantation of BMP2-treated dental pulp stem cell pellet culture onto the amputated pulp stimulated reparative dentin formation. These results prove that stem cell therapy has considerable promise in dentin regeneration. In near future, stem cells in combination with appropriate scaffolds and growth factors are materials, which may use for direct pulp capping.

In addition, multipotent stem cells were isolated from the remnant pulp of exfoliated deciduous teeth. It found that these stem cells are distinct from DPSCs with respect to their high proliferation rate, increases cell-population doublings, sphere-like cell-cluster formation, osteoinductive capacity in vivo, and failure to reconstitute a dentin-pulp like complex. It is indicate that deciduous teeth may be an ideal resource of stem cells to repair damage tooth structure, induce bone regeneration, and possibly to treat neural injury or degenerative diseases.

As we know, periodontal diseases can destroy the periodontal ligament, bone, and cementum. Destruction of this tissue is a cause of tooth loss. Recently, Seo et al. ${ }^{26}$ discovered stem cells from human periodontal ligament. These stem cells have the potential to generate periodontal ligament and cementum. In another research, mesenchymal stem cells have been used for periodontal defect treatment.
Hasegawa et al. ${ }^{22}$ was transplanted bone marrow-derived mesenchymal stem cell to experimental Class III periodontal defects. Four weeks after transplantation, the periodontal defects were almost regenerated with periodontal tissue. Cementoblasts, osteoblasts, osteocytes, and fibroblasts of the regenerated periodontal tissues were detected.

As a dentist, we frequently encounter defects in alveolar bone caused by trauma or inflammatory processes. Due to the high healing capacity of oral tissues, small defects frequently heal without major problems. However, if the affected area is large or complex tissues are involved, regeneration is generally incomplete. To regenerate the deteriorated tissues biologically based technique are required. Stem cell based-tissue engineering is a good alternative therapy. Several studies have already detailed the ability of MSCs transduced with BMP7 to elicit periodontal bone formation. ${ }^{27}$

Stem cell has a clinical potential for bone defect therapy. Until now, bone graft technique have been used for bone graft therapy. Autogenous bone graft from iliac bone make good healing, but this therapy is too expensive and morbidity. Furthermore, $8 \%$ of iliac graft make infection, nerve injury, blood loss, short and long-term pain and functional deficit. Recently, Ueda ${ }^{28}$ were able to demonstrate the transplantibility and therapeutic effects of MSC in bone defect. He formed MSCs transplantation in combination with biodegradable scaffold (beta TCP). The result of this research was the increased of bone regeneration in the defect.

Stem cells are also found in alveolar bone marrow. Isolated stem cells from alveolar bone marrow were cultured and expanded. These stem cells had potent osteogenic potential in vitro and in vivo. Based on the results, the researchers hope that transplantation of alveolar bone marrow stem cell can promote regeneration of alveolar bone in patients with periodontal diseases. ${ }^{15}$

In conclusion, stem cell present in dental pulp, periodontal ligament and alveolar bone marrow, and has a potential to repair and regenerate tooth and periodontal structures. Stem cells can be harvested from dental pulp, periodontal ligament, alveolar bone marrow, expanded, embedded in a appropriate scaffold, and transplanted back into defect to regenerate bone and tooth structures.

\section{REFERENCES}

1. Bonassar LJ, Vacanti CA. Tissue Engineering: the first decade and beyond. J Cell Biochem Supp 1998; 30(31): 297-303.

2. Nakashima M, Reddi H. The application of bone morphogenetic proteins to dental tissue engineering. Nature Biotechnology 2003; 21:1025-32.

3. Ballas CB, Zielske SP, Gerson S. Adult bone marrow stem cell and gene therapies: implications for greater use. J Cell Biochemistry Supp 2002; 38:20-8.

4. Caplan AI, Bruder SP. Mesenchymal stem cells: building blocks for molecular medicine in the $21^{\text {st }}$ century. Trends in Mol Med 2001; 7:259-64.

5. Pelled G, Turgeman G, Aslan H, Gazit Z, Gazit D. Mesenchymal stem cells for bone gene therapy and tissue engineering. Current Pharmaceutical Design, 2002; 8:1917-28. 
6. Rathjen PD, Lake J, Whyatt LM, Bettes MD, Ratjhen J. Properties and uses of embryonic stem cells: prospects for application to human biology and gene therapy. Reprod Fertil Dev 1998; 10:31-47.

7. Odorico JS, Kaufman DS, Thomson JA. Multilineage differentiation from human embryonic stem cell lines. Stem Cell 2001; 19:193-204

8. Kuehnle I, Goodell M. The therapeutic potential of stem cells from adults. BMJ 2002; 325:372-6.

9. Caplan AI. The mesengenic process. Clin Plastic Surg 1994; 21:42935 .

10. Makino S, Fukuda K, Miyoshi S, Kinoshi F, Kodama H, Pan J, Sano M, Takahashi T, Hori S, Abe H, Hata J, Umezawa A, Ogawa $\mathrm{S}$. Cardiomyocytes can be generated from marrow stromal cells in vitro. J Clin Invest 1999; 103:697-705.

11. Bianco P, Robey PG. Marrow stromal stem cells. J Clin Invest 2000; 105:1663-8.

12. Gronthos S, Mankani M, BrahimJ, Robey PG, Shi S. Postnatal human dental pulp stem cells (DPSCs) in vitro and in vivo. Proc Natl Acad Sci USA 2000; 97:13625-30.

13. Gronthos S, Brahim J, Li W, Fisher LW, Cherman N, Boyde A, DenBesten P, Robey PG, Shi S. Stem cell properties of human dental pulp stem cells. J Dent Rest 2002; 81:531-5.

14. Batouli S, Miura M, Brahim J, Tsutsui TW, Fisher LW, Gronthos S, Robey PG, Shi S. Comparison of stem-cell-medicated osteogenesis and dentinogenesis. J Dent Res 2003; 82:976-81.

15. Matsubara T, Suardita K, Ishii M, Sugiyama M, Igarashi A, Oda R, Nishimura M, Saito M, Nakagawa K, Yamanaka K, Miyazaki K, Shimizu M, Bhawal U, Tsuji K, Nakamura K, Kato Y. Alveolar bone marrow as a cell source for regenerative medicine: differences between alveolar and iliac bone marrow stromal cells. J Bone and Min. Res. 2005; 20: 399-409.

16. Miura M, Gronthos S, Zhao M, Lu B, Fisher LW, Robey PG, Shi S. SHED: stem cells from human exfoliated deciduous teeth. Proccedings of the National Academy of Science. 2003; 100: 5807-12.

17. Wakitani S, GotoT, Pineda SJ, Young RG, Mansour JM, Caplan AI, Goldberg VM. Mesenchymal cell-based repair of large, fullthicknesss defects of articular cartilage. J Bone Joint Surg Am 1994; 76:579-92.

18. Liu F, Aubin, JE, Malaval L. Expression of leukimia inhibitory factor (LIF)/interleukin-6 family cytokines and receptors during in vitro osteogenesis: differential regulation by dexamethasone and LIF. Bone 2002; 31:212-9.

19. Grande, DA, Pitman MI, Peterson J, Menche D, Klein M. The repair of experimentally produced defect in rabbit articularcartilage by autologous chondrocyte transplantation. J Orthop Res 1989; 7:208-18.

20. Shake JG, Gruber PJ, Baumgartner WA, Senechal G, Mryers J, Redmont JM, Pittenger MF, Martin BJ. Mesenchymal stem cell implantation I a swine myocardial infarct model: engraftment and functional effects. Ann Thorac Surg 2002; 73: 1919-26

21. Young RG, Butler DL, Weber W, Caplan AI, Gordon SL, Fink DJ. Use of mesenchymal stem cells in a collagen matrix for Achilles tendon repair. J Orthop Res 1998; 16: 406-13.

22. Hasegawa N, Kawaguchi H, Hirachi A, Takeda K, Mizuno N, Nishimura M, Koike C, Tsuji K, Iba H, KatoY, Kurihara H. Behavior of transplanted bone marrow-derived mesenchymal stem cells in periodontal defects. J Periodontol 2006; 77(6):1003-7.

23. Tziafas D, Smith AJ, Lesot H. Designing new treatment strategies in vital pulp therapy. J Dent 2000; 28: 77-92.

24. Cho MI, Garant PR. Development and general structure of the periodontium. Periodontol 2000; 24: 9-27.

25. Iohara K, Nakashima M, Ito M, Ishikawa M, Nakashima A, Akamine A. Dentin regeneration by dental pulp stem cell therapy with recombinant human bone morphogenetic protein 2. J Dent Res. 2004; 83(8): 590-5

26. Seo BM, Miura M, Gronthos S, Bartold PM, Batouli S, Brahim J, Young M, Robey PG, Wang CY, Shi S. Investigation of multipotent postnatal stem cells from human periodontal ligament. The Lancet 2004; 364:149-155.

27. Jin QM, Anusaksathien O, Webb SA, Rutherford RB, Giannobile WV. Gene therapy of bone morphogenic protein for periodontal tissue engineering. J Periodontol 2003; 74:202-13.

28. Ueda M. Maxillofacial bone regeneration using tissue engineering concepts. Dentistry in Japan 2003. 39:199-205 